\title{
RNA-seq analysis reveals extensive transcriptional plasticity to temperature stress in a freshwater fish species
}

\author{
Steve Smith ${ }^{1,3}$, Louis Bernatchez ${ }^{2}$ and Luciano B Beheregaray ${ }^{1 *}$
}

\begin{abstract}
Background: Identifying genes of adaptive significance in a changing environment is a major focus of ecological genomics. Such efforts were restricted, until recently, to researchers studying a small group of model organisms or closely related taxa. With the advent of next generation sequencing (NGS), genomes and transcriptomes of virtually any species are now available for studies of adaptive evolution. We experimentally manipulated temperature conditions for two groups of crimson spotted rainbowfish (Melanotaenia duboulayi) and measured differences in RNA transcription between them. This non-migratory species is found across a latitudinal thermal gradient in eastern Australia and is predicted to be negatively impacted by ongoing environmental and climatic change.

Results: Using next generation RNA-seq technologies on an Illumina HiSeq2000 platform, we assembled a de novo transcriptome and tested for differential expression across the treatment groups. Quality of the assembly was high with a N50 length of 1856 bases. Of the 107,749 assembled contigs, we identified 4251 that were differentially expressed according to a consensus of four different mapping and significance testing approaches. Once duplicate isoforms were removed, we were able to annotate 614 up-regulated transfrags and 349 that showed reduced expression in the higher temperature group.

Conclusions: Annotated blast matches reveal that differentially expressed genes correspond to critical metabolic pathways previously shown to be important for temperature tolerance in other fish species. Our results indicate that rainbowfish exhibit predictable plastic regulatory responses to temperature stress and the genes we identified provide excellent candidates for further investigations of population adaptation to increasing temperatures.
\end{abstract}

Keywords: Rainbowfish, Melanotaenia duboulayi, Transcriptomes, Climate change, Thermal adaptation

\section{Background}

The ability of species and populations to adapt to environmental change is the cornerstone of the emerging field of ecological genomics [1,2]. Until recently, genome-wide studies of genetic adaptation in non-model organisms were not possible. With the advent of massively parallel next generation sequencing technologies (NGS), these types of studies have become a reality [3] and while many of the challenges and preferred strategies are still being addressed [4-6], empirical studies are now starting to be reported [7-14]. Studies of transcriptome level responses to environmental change offer an opportunity

\footnotetext{
* Correspondence: Luciano.Beheregaray@flinders.edu.au

${ }^{1}$ Molecular Ecology Laboratory, School of Biological Sciences, Flinders

University, Adelaide, SA 5001, Australia

Full list of author information is available at the end of the article
}

to understand the underlying genetic basis for adaptation. Such studies represent a powerful approach to assessing the genes involved in adaptation to a changing climate, particularly increasing temperatures. By profiling transcriptional changes induced by temperature stress, it is possible to identify the gene regions or pathways that are likely to be the targets of selection. This information is crucial to enable researchers to assess levels of variation across these gene regions, at a landscape scale, to predict the capacity of organisms to adapt to a warming climate.

Genes involved in physiological adaptation to temperature stress have been uncovered in many species. Heat shock proteins [15], alcohol dehydrogenase [16] and lactate dehydrogenase genes [17] have all been shown to be related to heat tolerance. In fish, the list of candidates also includes many from other gene regions related to 
respiration and protein binding [18-20]. Apart from differences in coding regions, transcriptional regulation is also a source of variation that can potentially contribute to adaptive evolutionary change, particularly in the early stages of divergence. Studies in natural populations of gobies (Gillichthys mirabilis) have shown that short term exposure $\left(<8\right.$ hours) to a temperature of $32^{\circ} \mathrm{C}$ induces a strong upregulation of heat shock proteins (Hsps) in both gill and muscle tissues [21]. Many other transcripts related to a wide variety of biological processes including protein homeostasis, cell cycle control, cytoskeletal reorganisations, metabolic regulation, and signal transduction were differentially expressed in treatment and control groups. The majority of these genes displayed tissue-specific responses presumably related to the differing molecular functions associated with each tissue type. Logan and Somero [22] found that, with long-term acclimation to increased temperature (up to $28^{\circ} \mathrm{C}$ ), there was no upregulation of stress-related proteins and only slight, although detectable, differences in expression of genes involved in protein biosynthesis, transport and various metabolic categories. This they suggest indicates evidence of long-term acclimation showing a steady state condition involving relative energy costs for different processes. They later showed however, that stress related genes (HSP70, UBIQ, and $C D K N 1 B)$ were induced in long-term acclimatised fish subsequently exposed to acute heating conditions $\left(4^{\circ} \mathrm{C} /\right.$ hour) and that the onset temperature for significant expression change varied according to acclimation temperature [23]. Quinn et al. [24] also found increased expression of HSPs and Ubiquitin in Arctic charr (Salvelinus alpinus) exposed to temperature stress and reported a down regulation of haemoglobin genes in fish that showed tolerance to increased temperatures. Another cold climate fish, Trematomus bernacchii, has been shown to be unable to mount a heat shock response despite retaining the heat shock gene $H s p 70$ and the regulation factor HSF1 [25]. Further work showed that many other genes associated with the cellular stress response were induced by heat stress. The inability to mount a heat shock response however, highlights the susceptibility of this species to global warming and raises the question as to how this and other species will be able to adapt to increasing temperatures.

Buckley and Hofmann [26] examined the extensive plasticity in $\mathrm{Hsp}$ induction in gobies acclimatised to different thermal backgrounds $\left(13^{\circ} \mathrm{C}, 21^{\circ} \mathrm{C}\right.$, and $\left.28^{\circ} \mathrm{C}\right)$. They found that the activation temperature of the transcriptional regulator HSF1 was positively associated with the acclimatisation temperature indicating that plasticity in heat shock response is linked to plasticity in the regulatory framework governing Hsps. While adaptive plasticity is often seen as a mechanism that can slow or dampen divergent selection, it has been argued that it can also lead to rapid speciation if there are strong correlations between phenotype and environment combined with significant population structure [27]. By examining the transcriptomic response to temperature stress we can develop a better understanding of the genes and biochemical pathways that are fundamental to physiological acclimatisation to a warming environment and gain insights into the regulatory changes that accompany adaptation over evolutionary timescales [28].

Australian rainbowfish are an ideal species group to test hypotheses about the genetic responses to increasing temperatures. In particular, the crimson-spotted rainbowfish (Melanotaenia duboulayi) is a subtropical freshwater species found along a north-south temperature gradient in eastern Australia. Their distribution ranges over several ecoregions which, coupled with a strong population structure and local abundance [29-31], makes them a well suited model for studying local adaptation. The ease of maintaining captive populations of rainbowfish also make them amenable to a range of laboratory-based experimental studies [32-34]. In this study, we maintained groups of M. duboulayi at ambient and elevated temperature levels and then used an RNA-seq approach to assess transcriptome level changes related to temperature stress. Our aim is to provide an initial investigation of the transcriptomic response to thermal stress in rainbowfish. As such, this will allow for the screening of many more individuals via genotyping of candidate SNPs. In addition we present the first annotated transcriptome and gene catalogue for the order Atheriniformes. Our goal is to identify key candidate genes and make a first step towards understanding the important biochemical pathways on which selection is likely to act in a warming climate.

\section{Methods}

\section{Source of fish and design of temperature trial}

Crimson spotted rainbowfish were collected using a handnet from a location in the upper reaches of the Brisbane River, near the township of Fernvale $\left(27^{\circ} 26^{\prime} 37.39^{\prime \prime} \mathrm{S}\right.$, $\left.152^{\circ} 40^{\prime} 12.76^{\prime \prime E}\right)$. Water monitoring data from the Queensland Department of Environment and Resource Monitoring (DERM) show the average daily mean temperatures for this location ranged between $12.2^{\circ} \mathrm{C}$ in winter and $28.3^{\circ} \mathrm{C}$ in summer from January $1^{\text {st }} 2004$ to January $1^{\text {st }} 2011$ (http://watermonitoring.derm.qld.gov.au). Fish were transported live to Flinders University animal rearing facility and acclimatised at a temperature of $21^{\circ} \mathrm{C}$ for a period of 30 days prior to the start of the temperature trials. For the trials we used only adult male rainbowfish of about the same length (a proxy for age), since gender and age can affect expression responses [35]. These individuals were randomly assigned to a treatment or a control group ( $n=6$ per group). Temperature in the 
treatment group was increased by $2^{\circ} \mathrm{C}$ per day over a period of six days towards a target of $33^{\circ} \mathrm{C}$. This target represents the projected average summer temperature for this region in 2070 based on a high emission scenario of the International Panel on Climate Change: http://www.climatechangeinaustralia.gov.au/qldtemp15. php. This temperature condition was then maintained for 14 days. The control group was kept at $21^{\circ} \mathrm{C}$ for the duration of the experiment. All animal handling was performed in accordance with the Australian Code of Practice for the Care and Use of Animals for Scientific Purposes, 2004 and approved by the Flinders University Animal Welfare Committee (AWC E342).

\section{RNA extraction, Illumina library preparation and sequencing}

Upon completion of the temperature trial, fish were sacrificed using AQUI-S ${ }^{\bullet}$ solution [36] and dissected immediately to remove their livers. Although increased temperature has been shown to differentially induce expression changes in different tissue types [21,37], we were restricted to examining just one tissue type due to logistical constraints. We selected liver due to previous research linking this tissue type to heat stress responses [38-40]. Total RNAs were individually extracted using the Ambion Magmax ${ }^{\mathrm{Tm}}-96$ total RNA isolation kit (Life Sciences) according to the manufacturer's instructions. Briefly, $5 \mathrm{mg}$ of tissue was placed in the lysis solution and homogenised in Qiagen Tissuelyzer ${ }^{\mathrm{TM}}$ for a period of 30 sec. Nucleic acids were captured onto magnetic beads, washed and treated with DNase. Total RNA was then eluted in $50 \mu \mathrm{l}$ elution buffer. RNA quality and concentration was measured using an RNA Pico chip on an Agilent Bioanalyzer. Normalised starting quantities of total RNA were then used to prepare 12 separate Illumina sequencing libraries with the TruSeq ${ }^{\mathrm{Tm}}$ RNA sample preparation kit (Illumina). Library preparation was performed as per the manufacturer's instructions. In the final step before sequencing, all 12 individual libraries were normalised and pooled together using the adapter indices supplied by the manufacturer (Illumina MID tags $2,4-7,12-16,18,19)$. Pooled sequencing was then performed as $101 \mathrm{bp}$, paired-end reads in a single lane of an Illumina HiSeq2000 instrument housed at the Ramaciotti Centre for Gene Function Analysis, University of New South Wales.

\section{Quality control and de novo assembly}

Sequence data were sorted by individual and adapters were trimmed by the service provider prior to analysis. Quality filtering was performed using the FastX-toolkit suite of pre-processing tools (http://hannonlab.cshl.edu/ fastx_toolkit/index.html) in a Galaxy setting [41]. Based on the FastX quality statistics, the first two and last 5 bases were trimmed from each read as they had consistently low phred scores $(<\mathrm{Q} 15)$. Paired reads were then joined and a quality filter applied such that any combined reads having $<90 \%$ of bases with a phred score of Q20 or higher were discarded. Paired reads were then split and interleaved to suit the input style of the de novo assembly program. Transcriptome assembly was performed de novo with the program Velvet/Oases [42]. This program reconstructs independent assemblies based on different k-mer values used to build a de Bruijn graph. The program then uses dynamic error removal adapted to RNA-seq data and implements a robust scaffolding method to predict full length transfrags. Multiple single k-mer assemblies are then merged to cover genes at different expression levels without redundancy. Two individuals from each of the treatment and control groups were pooled as input for the assembly. Assemblies were compiled for a k-mer range of 19 to 49 with an expected insert size between paired ends of $300 \mathrm{bp}$ and a coverage cut-off value set to 4.2. We tested different merged assembly ranges based on the summary statistics for each individual k-mer assembly [43]. The outcome of each merge was assessed with respect to the optimal assembly parameters [4]. The optimal assembly should achieve the best balance between large median, mean and N50 contig lengths while minimising the total number of contigs but maintaining a large summed contig length. As Oases is vulnerable to misassembly at low k-mer values, we adopted a conservative approach of merging $\mathrm{k}$-mer values $>\mathrm{k}=19$. Optimal assembly was achieved with a k-mer range of 19 to 41 .

\section{Mapping of sequence reads and differential expression analysis}

To test for differential expression (DE), individual sequence reads for each sample were mapped back to the assembled transcriptome with the alignment program Bowtie [44]. Bowtie was implemented in the $-\mathrm{v}$ alignment mode with the maximum number of mismatches set to 3 . Paired end reads were aligned to the transcriptome with both read pairs needing a valid alignment within a given locus to be counted as a match. If more than one alignment was possible the best match was reported according to the least number of mismatches for each read and overall for the pair. The reproducibility of the alignment approach was tested by performing the mapping step with BWA, an alternative alignment program [45]. The number of reads aligning to each transfrag for each sample was calculated with the IdxStats command of Samtools [46]. Count data was then used as input for the program DESeq [47] which estimates variance-mean dependence in the data and tests for differential expression based on the negative binomial distribution. The six samples from each treatment were used to generate mean expression levels with associated variances. Differential 
expression was tested at a significance level of $\alpha=0.05$ adjusted to match a $5 \%$ false discovery rate using the Benjamini-Hochberg procedure. The threshold for foldchange differences is determined by the significance testing as the power to detect significant differential expression depends on the expression strength. For weakly expressed genes, stronger changes are required for the gene to be called significantly expressed. We also compared DE methodology by running the EdgeR program to assess significant differences in the count data. A consensus list of DE genes was then generated from the four analysis approaches adopted (i.e. Bowtie-DESeq, Bowtie-EdgeR, BWA-DESeq, BWA-EdgeR). Significantly up and down regulated transfrags were selected and blasted against the NCBI database using blastx in the program Blast2GO [48]. Blastx was performed against the NCBI nucleotide database with the minimum E-value score set to $1.0 \mathrm{E}-06$. To assign gene ontology terms to each annotated sequence, successful blast hits were mapped and annotated using Blast2GO for the entire assembled transcriptome with the annotation cut-off threshold set to 55 and the GO level weighting set to 5 .

\section{Results and discussion}

Raw sequencing data and quality statistics

The single lane of Illumina HiSeq2000 produced close to 128 million paired-end reads $(2 \times 101 \mathrm{bp})$. After trimming and quality filtering, $12.3 \%$ of reads were discarded leaving over 224 million reads for downstream analysis $(2 \times 94 \mathrm{bp})$. The final number of reads per individual ranged from 11.7 million to 29 million (mean $=18.7$ million \pm 1.4 million). The number of reads in each treatment group was well balanced with 112.3 million in the $21^{\circ} \mathrm{C}$ group and 112.0 million in the $33^{\circ} \mathrm{C}$ group (Additional file 1: Table S1). We selected the best k-mer merge range for assembly based on the distribution of assembly statistics for the individual k-mer assemblies from $\mathrm{k}=19$ to $\mathrm{k}=49$ (see Table 1 ). The merged assembly from a k-mer range of 21 to 39 scored best on the balance of these parameters with a N50 value of 1,856 and a total number of contigs of 107,749 . While this range may exclude some rare, low-abundant transcripts, it presents a more conservative and reliable approach to differential expression testing by emphasising the accuracy of the assembly rather than the identification of lowabundant transcripts from both treatments. Annotation of the transfrags with the Blast2Go software suite resulted in 65,105 (60.4\%) blast hits and 53,278 (49.4\%) successfully annotated sequences.

\section{Differential expression analyses}

The four different combinations of mapping and DE testing produced vastly different numbers of DE transfrags (see Table 1, Figure 1). The combination of BWA alignment followed by EdgeR DE analysis identified the most with 14,076 DE transfrags, whereas Bowtie followed by DESeq identified the least with 5,577 (Figure 1). The difference between the approaches likely arises from the different characteristics of the two aligners combined with the sensitivities of the DE tests. Bowtie does not allow gapped alignments and makes use of the base quality scores [49], making it more conservative than BWA in the number of mapped reads. On the other hand DESeq has also been shown to be more conservative than EdgeR when identifying DE genes from low count data [50] which likely explains the lower number of hits in multiplex sequencing strategies such as ours. The total number of DE transfrags identified by all four approaches was 4,251 (Figure 2). We adopted a conservative approach and selected only these transfrags to blast against the reference database. Future RNA-seq studies should assess their priorities for DE gene discovery and select the detection strategy based upon the need for identifying lowly expressed genes versus the accuracy expected given the number of replicates used [51]. Robles et al. [50] showed that EdgeR could be used to detect higher numbers of DE transfrags from low count data without compromising accuracy when the number of biological replicates was at least six in each treatment group.

The Blast2GO program was able to find sequence similarities for 2,740 of the DE transfrags but could not find mapping or annotation information for a further 634 of them, leaving 2,106 DE transfrags which were successfully annotated. The top 15 matching species from the BLAST query were all fish species with the most BLAST hits being for the Nile tilapia Oreochromis niloticus with 583 matches. Duplicate gene isoforms were detected by matching identical annotated gene names from the Blast2GO output. These isoforms were then combined and reported as single "genes". Once isoforms were combined, there were 614 genes that were up-regulated in the high temperature treatment with 349 genes being down-regulated (see Additinal file 1: Table S2a and b). For significantly down-regulated transfrags, the mean fold-change between ambient and high-temperature conditions was 4.0-fold, with a range from 55.6-fold for $\mathrm{g} 2 / \mathrm{m}$ phase specific e3 ubiquitinprotein ligase to 2.2-fold for the Phytanoyl-peroxisomallike protein. The mean fold-change for significantly up-regulated transfrags was 11.13 , ranging from 1.98 (for the cyclin-dependent kinase 2 interacting protein) to 259-fold (for the Heat shock protein Hsp-90-like).

\section{Ontology of differentially expressed genes}

Many functional classes of genes were affected by temperature stress. As expected, heat shock protein genes including HSPA4 (12.3 x), Hsp60 (6.6 x), Hsp70 $(9.9 \mathrm{x})$ and $H \operatorname{sp} 90 \alpha(141.3 \mathrm{x})$ were significantly up- 
Table 1 Assembly statistics for k-mer lengths 19-49 and different k-mer merge ranges from the Oases de novo assembly program

\begin{tabular}{|c|c|c|c|c|c|c|c|c|c|c|}
\hline & k19 & k21 & $k 23$ & $k 25$ & k27 & k29 & k31 & k33 & k35 & k37 \\
\hline Total sequences & $1.2 \mathrm{E}+05$ & $7.3 \mathrm{E}+04$ & $6.2 \mathrm{E}+04$ & $5.5 \mathrm{E}+04$ & $5.2 \mathrm{E}+04$ & $5.0 \mathrm{E}+04$ & $4.8 \mathrm{E}+04$ & $7.0 \mathrm{E}+04$ & $8.2 \mathrm{E}+04$ & $8.1 \mathrm{E}+04$ \\
\hline Total bases & $6.7 \mathrm{E}+07$ & $6.1 \mathrm{E}+07$ & $5.6 \mathrm{E}+07$ & $5.3 \mathrm{E}+07$ & $5.1 \mathrm{E}+07$ & $5.0 \mathrm{E}+07$ & $4.9 \mathrm{E}+07$ & $6.2 \mathrm{E}+07$ & $7.2 \mathrm{E}+07$ & $7.3 \mathrm{E}+07$ \\
\hline Min sequence length & $7.1 \mathrm{E}+01$ & $1.0 \mathrm{E}+02$ & $8.1 \mathrm{E}+01$ & $1.0 \mathrm{E}+02$ & $9.8 \mathrm{E}+01$ & $1.0 \mathrm{E}+02$ & $9.0 \mathrm{E}+01$ & $1.0 \mathrm{E}+02$ & $1.0 \mathrm{E}+02$ & $1.0 \mathrm{E}+02$ \\
\hline Max sequence length & $1.5 \mathrm{E}+04$ & $1.7 \mathrm{E}+04$ & $2.0 \mathrm{E}+04$ & $1.8 \mathrm{E}+04$ & $2.1 \mathrm{E}+04$ & $2.3 \mathrm{E}+04$ & $1.8 \mathrm{E}+04$ & $1.2 \mathrm{E}+04$ & $1.3 \mathrm{E}+04$ & $1.3 \mathrm{E}+04$ \\
\hline Average sequence length & 558.04 & 837.19 & 906.27 & 960.63 & 979.44 & 991.21 & 1010.47 & 884.15 & 888.33 & 901.83 \\
\hline Median sequence length & 356 & 527 & 546 & 580 & 584 & 590 & 605 & 595 & 583 & 584 \\
\hline N50 length & 873 & 1397 & 1585 & 1686 & 1746 & 1759 & 1801 & 1398 & 1460 & 1493 \\
\hline$(A+T) s$ & $55.25 \%$ & $55.32 \%$ & $55.25 \%$ & $55.16 \%$ & $55.16 \%$ & $55.17 \%$ & $55.12 \%$ & $55.07 \%$ & $55.21 \%$ & $55.35 \%$ \\
\hline$(G+C) s$ & $43.99 \%$ & $44.27 \%$ & $44.47 \%$ & $44.60 \%$ & $44.64 \%$ & $44.67 \%$ & $44.72 \%$ & $44.83 \%$ & $44.63 \%$ & $44.49 \%$ \\
\hline Ns & $0.77 \%$ & $0.41 \%$ & $0.28 \%$ & $0.23 \%$ & $0.21 \%$ & $0.16 \%$ & $0.16 \%$ & $0.10 \%$ & $0.16 \%$ & $0.16 \%$ \\
\hline
\end{tabular}


Table 1 Assembly statistics for k-mer lengths 19-49 and different k-mer merge ranges from the Oases de novo assembly program (Continued)

\begin{tabular}{|c|c|c|c|c|c|c|c|c|c|c|}
\hline & k39 & k41 & k43 & k45 & k47 & k49 & k19_39 & k21_39 & k25_47 & k21_49 \\
\hline Total sequences & $8.2 \mathrm{E}+04$ & $4.3 \mathrm{E}+04$ & $4.1 \mathrm{E}+04$ & $4.0 \mathrm{E}+04$ & $3.9 \mathrm{E}+04$ & $5.6 \mathrm{E}+04$ & $4.5 \mathrm{E}+05$ & $1.1 \mathrm{E}+05$ & $4.0 \mathrm{E}+05$ & $4.9 \mathrm{E}+05$ \\
\hline Total bases & $7.2 \mathrm{E}+07$ & $4.4 \mathrm{E}+07$ & $4.3 \mathrm{E}+07$ & $4.2 \mathrm{E}+07$ & $4.1 \mathrm{E}+07$ & $5.1 \mathrm{E}+07$ & $4.8 \mathrm{E}+08$ & $1.3 \mathrm{E}+08$ & $4.4 \mathrm{E}+08$ & $5.5 \mathrm{E}+08$ \\
\hline Min sequence length & $1.0 \mathrm{E}+02$ & $9.9 \mathrm{E}+01$ & $1.0 \mathrm{E}+02$ & $1.0 \mathrm{E}+02$ & $1.0 \mathrm{E}+02$ & $1.0 \mathrm{E}+02$ & $1.0 \mathrm{E}+02$ & $1.0 \mathrm{E}+02$ & $1.0 \mathrm{E}+02$ & $1.0 \mathrm{E}+02$ \\
\hline Max sequence length & $1.4 \mathrm{E}+04$ & $1.7 \mathrm{E}+04$ & $1.7 \mathrm{E}+04$ & $1.7 \mathrm{E}+04$ & $1.7 \mathrm{E}+04$ & $1.4 \mathrm{E}+04$ & $2.3 \mathrm{E}+04$ & $1.7 \mathrm{E}+04$ & $2.3 \mathrm{E}+04$ & $2.3 \mathrm{E}+04$ \\
\hline Average sequence length & 884.62 & 1026.19 & 1037.21 & 1042.06 & 1049.7 & 903.57 & 1071.74 & 1245.3 & 1114.01 & 1124.62 \\
\hline Median sequence length & 568 & 624 & 634 & 639 & 650 & 608 & 780 & 930 & 805 & 818 \\
\hline N50 length & 1492 & 1785 & 1789 & 1795 & 1786 & 1457 & 1589 & 1856 & 1671 & 1689 \\
\hline$(A+T) s$ & $55.38 \%$ & $55.04 \%$ & $55.03 \%$ & $55.05 \%$ & $55.03 \%$ & $54.85 \%$ & $55.95 \%$ & $55.11 \%$ & $55.69 \%$ & $56.13 \%$ \\
\hline$(G+C) s$ & $44.48 \%$ & $44.86 \%$ & $44.87 \%$ & $44.87 \%$ & $44.89 \%$ & $45.08 \%$ & $44.05 \%$ & $44.89 \%$ & $44.31 \%$ & $43.87 \%$ \\
\hline Ns & $0.14 \%$ & $0.10 \%$ & $0.10 \%$ & $0.08 \%$ & $0.08 \%$ & $0.06 \%$ & $0.00 \%$ & $0.00 \%$ & $0.00 \%$ & $0.00 \%$ \\
\hline
\end{tabular}




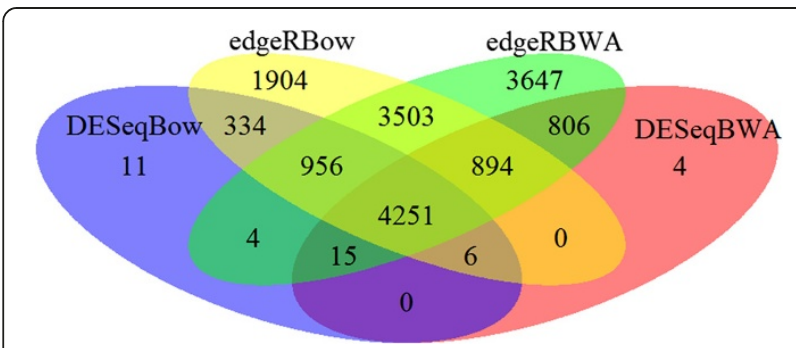

Figure 1 Overlap between the number of differentially expressed transfrags detected from the four combinations of mapping and significance testing methods for sequences involved in transcriptomic response to increased temperature for the rainbowfish Melanotaenia duboulayi. See text for details of mapping and testing methods used.

regulated in heat stressed fish. These transcripts are well characterised as stress inducible and have been shown, in many species, to be involved in protection against apoptosis or as a molecular chaperone under extended exposure to heat stress $[15,19,20,52-56]$. Further to these well-characterised stress related genes, the gene ontology analysis also identified transcripts involved in catabolism (11\% of annotated sequences) and lipid metabolism (12\% of annotated sequences) as being the important biological processes in the response to temperature stress (Figure 3a). As with other studies in fish, regulation of metabolic processes are clearly important parts of the heat stress response $[21,22,24]$. A large proportion of the individual over-expressed genes in rainbowfish were related to oxidoreductase activity, mitochondrial components and organelle membranes. These gene categories are typically associated with increased metabolism, particularly

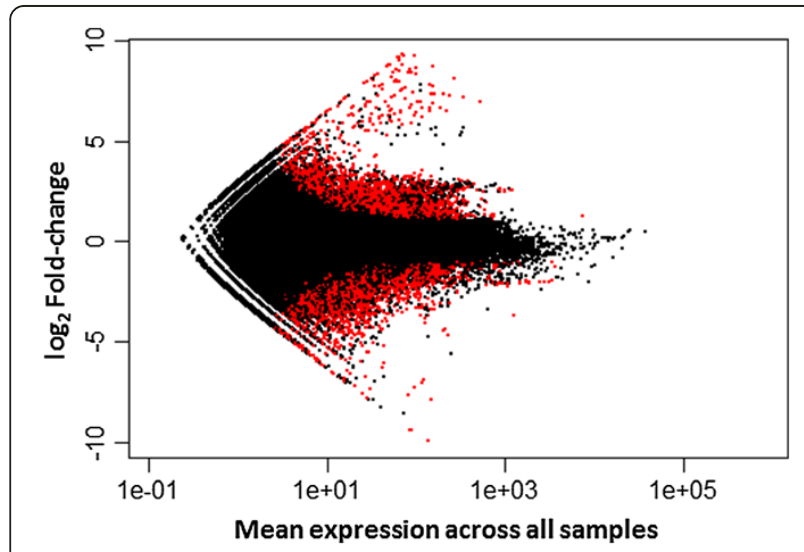

Figure 2 Differential expression of 107,749 transfrags assembled for the rainbowfish Melanotaenia duboulayi under different temperature treatments $\left(21^{\circ} \mathrm{C}\right.$ vs. $\left.33^{\circ} \mathrm{C}\right)$. Results are shown as the $\log _{2}$ fold change in expression versus the mean expression level between treatment groups. Red dots above zero fold change represent significantly up-regulated transfrags whereas red dots below zero fold change represent significantly downregulated tranfrags at the 0.5 false discovery rate. to cope with increased temperature and the related hypoxic conditions. Additionally we found a role for genes of the ubiquitin family and the gene $78 \mathrm{kDa}$ glucoseregulated protein precursor which, similar to Quinn et al. [57], were upregulated in response to heat stress. Gene ontology analysis also identified biomolecular binding and catalytic activity as the major molecular functions affected by exposure to different temperature regimes (see Figure 3b). Within these broad categories, protein binding and ATP binding were the major biomolecular binding functions affected by differentially expressed transfrags with node scores of 244 and 226 respectively. For catalytic activity, transferase activity (nodescore $=53$ ) and oxidoreductase activity were prominent (node score $=54$ ). These functional categories, combined with electron carrier activity (node score $=63$ ), is congruent with the expected role of aerobic respiration in response to the increased temperature.

While the Hsp genes are commonly identified as overexpressed in short-term temperature manipulation experiments [24,37], they are less likely to be targets for selection during gradual temperature shifts associated with climate change [22,53]. Hsp genes represent a physiological response to sudden stressors and therefore plasticity in these traits is unlikely to be adaptive over longer timescales [58]. The more likely candidates for an adaptive genetic response are those genes involved in what has been termed the "cellular homeostatis response" to long-term temperature stress [59]. Unlike stress response genes that provide an immediate early response to macromolecular damage and sudden changes in cellular redox potential, the cellular homeostatasis response involves effector proteins mediating parameter specific adaptation to environmental change.

\section{Responses associated with prolonged exposure to heat stress}

Prolonged exposure to increased temperatures has previously been associated with gene ontologies related to protein folding, oxidative stress and immune function $[18,19]$. Similarly, we detected significant upregulation of genes with these ontologies in the high temperature treatment such as Calnexin $(2.8 \mathrm{x}), \mathrm{NADH}$ dehydrogenase $(2.5 \mathrm{x})$, and glutathione $\mathrm{S}$-transferase $(5.1 \mathrm{x})$ suggesting long-term reallocation of energy resources. Plasticity in the expression of these genes is more likely to be adaptive and allow localised populations to survive in a changing environment, eventually leading to divergent selection. Kassahn et al. [53] grouped stress-response transcripts into four different clusters according to the pattern of regulation detected under short versus long-term exposure to heat stress. They suggested that long-term exposure to heat stress in a coral reef fish $\left(31^{\circ} \mathrm{C}\right.$ for five days) induces expression of genes involved in development and immune 
(a)

\section{Biological Process}

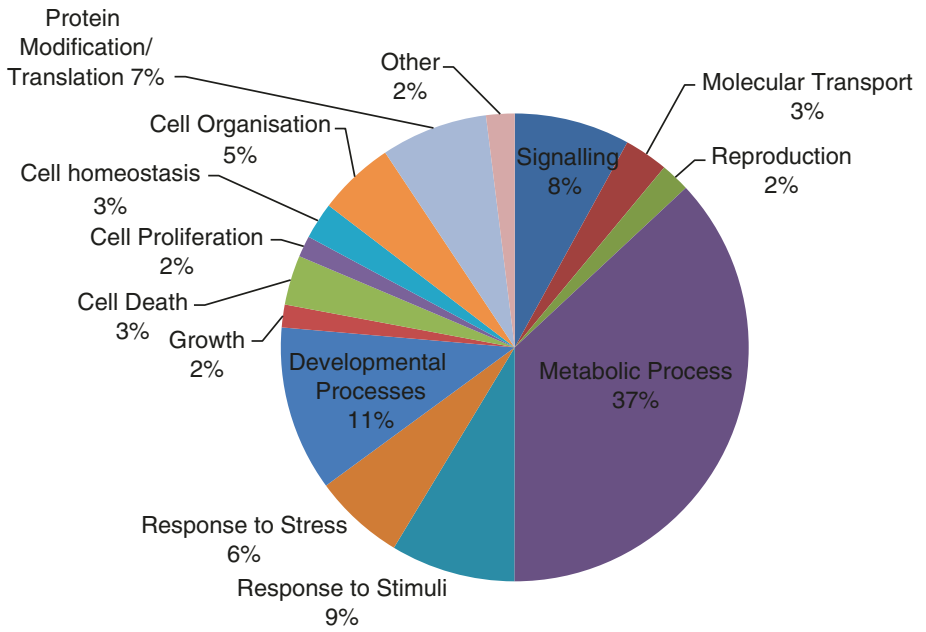

(b)

\section{Molecular Function}

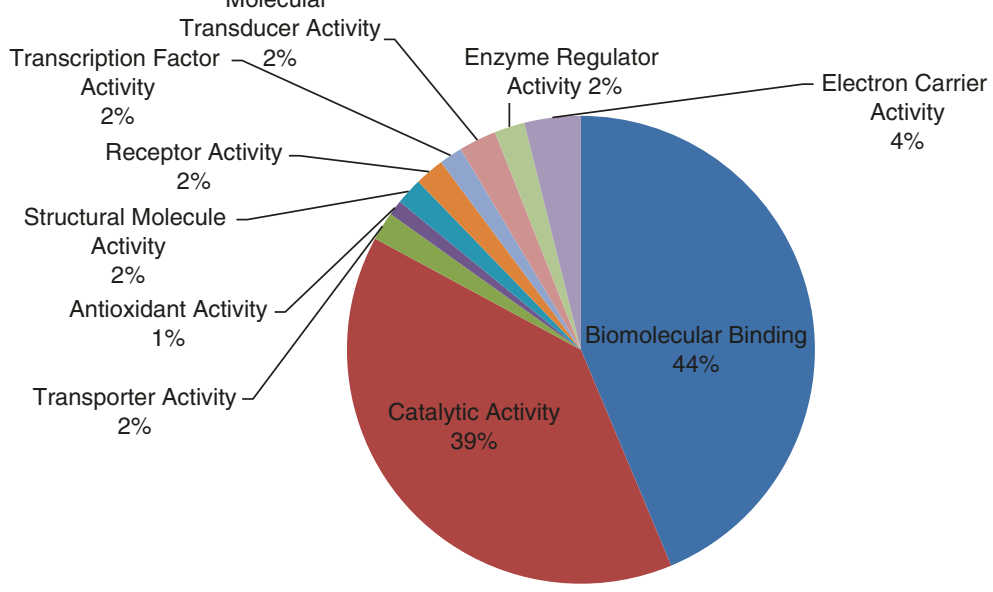

(c) Cellular Component

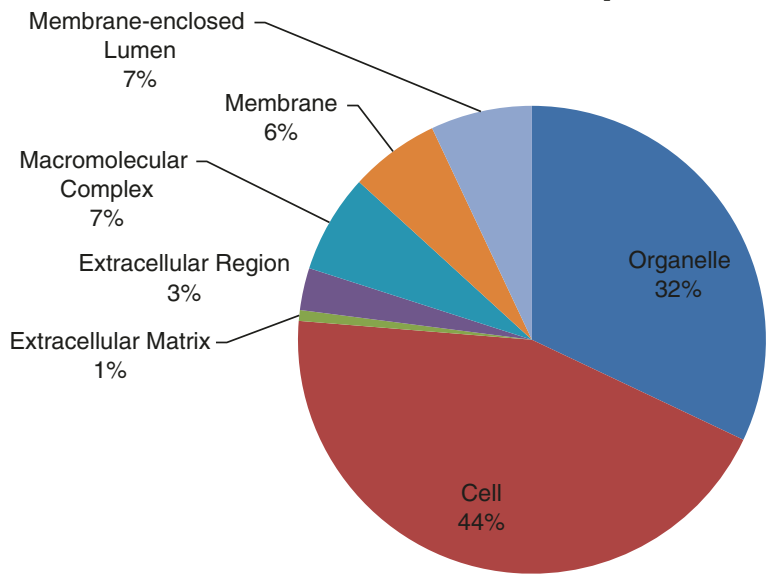

Figure 3 Distribution of annotated transfrags assigned to (a) biological processes or (b) molecular functions or (c) the cellular components according to gene ontology association. Analysis carried out with the Blast2Go program for sequences involved in transcriptomic response to increased temperature for the rainbowfish Melanotaenia duboulayi. 
Table 2 Candidate genes for broad scale studies of temperature response in the crimson spotted rainbowfish, Melanotaenia duboulayi

\begin{tabular}{|c|c|c|c|}
\hline Seq. description & min. e value & Fold change & Primary gene Ontology \\
\hline 3-keto-steroid reductase-like & $5.09 \mathrm{E}-49$ & 2.25 & C:endoplasmic reticulum membrane \\
\hline hydroxymethylglutaryl- cytoplasmic & $9.30 \mathrm{E}-32$ & 2.25 & P:isoprenoid biosynthetic process \\
\hline smooth muscle cell-specific protein sm22 alpha & $1.44 \mathrm{E}-126$ & 2.28 & P:muscle organ development \\
\hline phospholemman precursor & $1.98 \mathrm{E}-44$ & 2.32 & C:membrane \\
\hline ap-2 complex subunit sigma & $1.34 \mathrm{E}-91$ & 2.32 & P:axon guidance \\
\hline acyl carrier mitochondrial precursor & 1.87E-82 & 2.34 & F:phosphopantetheine binding \\
\hline nadh-cytochrome b5 reductase 2 & $3.52 \mathrm{E}-137$ & 2.34 & F:cytochrome-b5 reductase activity \\
\hline sterol-4-alpha-carboxylate 3- decarboxylating-like & $7.81 \mathrm{E}-44$ & 2.36 & P:steroid biosynthetic process \\
\hline retinol-binding protein cellular & $3.29 \mathrm{E}-86$ & 2.38 & P:transport \\
\hline ribosomal rna processing protein 36 homolog & $9.82 \mathrm{E}-58$ & 2.38 & C:nucleolus \\
\hline protein cdv3 homolog & 4.64E-64 & 2.38 & C:cytoplasm \\
\hline nadh dehydrogenase 1 alpha subcomplex subunit 6 & $1.63 \mathrm{E}-77$ & 2.41 & C:mitochondrial inner membrane \\
\hline y chain e2 ubiquitin-hect & $1.04 \mathrm{E}-45$ & 2.41 & P:endosome transport \\
\hline monoamine oxidase & 0 & 2.42 & P:catecholamine metabolic process \\
\hline small nuclear ribonucleoprotein $\mathrm{sm} \mathrm{d} 2$ & $2.44 \mathrm{E}-64$ & 2.42 & P:ncRNA metabolic process \\
\hline transketolase & $6.63 \mathrm{E}-137$ & 2.42 & F:transketolase activity \\
\hline rho-class glutathione s-transferase & 1.95E-101 & 2.43 & F:transferase activity \\
\hline thioredoxin domain-containing protein 14 precursor & $2.31 \mathrm{E}-72$ & 2.44 & P:cell redox homeostasis \\
\hline ubiquitin-conjugating enzyme e2 variant 2 & $1.43 \mathrm{E}-85$ & 2.45 & F:acid-amino acid ligase activity \\
\hline coiled-coil domain-containing protein 47 precursor & 0 & 2.46 & P:embryonic development \\
\hline ubiquinol-cytochrome c reductase core protein ii & 0 & 2.47 & F:metalloendopeptidase activity \\
\hline 3-hydroxy-3-methylglutaryl-coenzyme a synthase 1 & $8.22 \mathrm{E}-40$ & 2.99 & P:response to tellurium ion \\
\hline nuclear factor erythroid derived 2-like 1 & $2.00 \mathrm{E}-60$ & 3.24 & P:heme biosynthetic process \\
\hline glutathione s-transferase & $3.32 \mathrm{E}-30$ & 5.07 & F:glutathione transferase activity \\
\hline cyclin-dependent kinase inhibitor 1 & $6.69 \mathrm{E}-65$ & 5.86 & P:cellular response to stimulus \\
\hline catechol-o-methyltransferase domain-containing protein 1 & 1.45E-75 & -2.44 & F:O-methyltransferase activity \\
\hline period homolog 3 & $1.01 \mathrm{E}-173$ & -2.44 & C:cytoplasm \\
\hline histamine n-methyltransferase & 1.19E-130 & -2.50 & P:respiratory gaseous exchange \\
\hline scinderin like a & 0 & -2.50 & P:eye development \\
\hline 5-aminolevulinate erythroid- mitochondrial-like & 0 & -2.50 & P:response to hypoxia \\
\hline 55 kda erythrocyte membrane protein & $2.00 \mathrm{E}-91$ & -2.50 & C:intracellular non-membrane-bounded organelle \\
\hline plakophilin 3 & 0 & -2.50 & F:binding \\
\hline cbp p300-interacting transactivator 3b & 5.19E-44 & -2.50 & C:nucleus \\
\hline lysosomal alpha-glucosidase-like & 0 & -2.56 & F:carbohydrate binding \\
\hline actin-binding lim protein 1 long isoform isoform cra_a & $4.22 \mathrm{E}-35$ & -2.56 & P:axon guidance \\
\hline udp-glucuronosyltransferase 2a2-like isoform 2 & $1.10 \mathrm{E}-93$ & -2.56 & F:transferase activity, transferring hexosyl groups \\
\hline glucose-fructose oxidoreductase domain-containing protein 1-like & 4.30E-38 & -2.56 & C:extracellular region \\
\hline dual specificity tyrosine-phosphorylation-regulated kinase $1 \mathrm{~b}$ & 0 & -2.56 & P:protein amino acid autophosphorylation \\
\hline synaptobrevin homolog ykt6 & $3.21 \mathrm{E}-121$ & -2.56 & C:Golgi membrane \\
\hline serine-pyruvate mitochondrial precursor & $2.50 \mathrm{E}-25$ & -2.56 & P:metabolic process \\
\hline transmembrane protein 192 & $6.58 \mathrm{E}-112$ & -2.63 & C:membrane \\
\hline protein creg2-like & $9.34 \mathrm{E}-142$ & -2.63 & C:cytoplasmic part \\
\hline ras-related protein rab-13-like & $1.31 \mathrm{E}-67$ & -2.63 & P:vesicle-mediated transport \\
\hline c-jun-amino-terminal kinase-interacting protein 4 isoform partial & 0 & -2.63 & F:protein binding \\
\hline histone-lysine n-methyltransferase setd3-like & $3.00 \mathrm{E}-148$ & -2.63 & P:peptidyl-lysine monomethylation \\
\hline peroxisome proliferator-activated receptor alpha & $2.04 \mathrm{E}-78$ & -2.86 & P:steroid hormone mediated signaling pathway \\
\hline
\end{tabular}


Table 2 Candidate genes for broad scale studies of temperature response in the crimson spotted rainbowfish, Melanotaenia duboulayi (Continued)

\begin{tabular}{llll}
\hline cytochrome p450 1a & $5.02 \mathrm{E}-152$ & -3.70 & C:endoplasmic reticulum membrane \\
\hline thyrotrophic embryonic factor & $9.16 \mathrm{E}-157$ & -3.85 & P:cellular response to light stimulus \\
\hline nuclear receptor subfamily 1 group d member 2 & $3.54 \mathrm{E}-102$ & -4.17 & P:steroid hormone mediated signaling pathway \\
\hline vitellogenin ab & 0 & -10.0 & F:lipid transporter activity \\
\hline
\end{tabular}

Genes correspond to transfrags with mid-range differential expression values that match metabolic, developmental, or immune response processes from gene ontology assignment by the Blast2Go program. Gene ontology abbreviations: $\mathrm{P}=$ biological process, $\mathrm{F}=$ molecular function, $\mathrm{C}=$ cellular component.

function whereas genes related to metabolic function are suppressed. Our data, from long-term exposure to heat stress in rainbowfish $\left(33^{\circ} \mathrm{C}\right.$ for 14 days), support those findings. Developmental processes and metabolic processes accounted for $48 \%$ of dysregulated transfrags (Figure 3a). Immune function seems less important in our dataset and is covered by the "response to stimuli" category representing $9 \%$ of DE transfrags including the natural killer cell enhancement factor (upregulated $2.8 \mathrm{x}$ ). It is possible that the longer exposure to heat stress in our study allowed recovery from the immediate activation of the immune function genes.

Under simulated models of divergence with plasticity, selection is possible when plasticity is moderate, dispersal ability is low and there are no fitness costs to plasticity [60]. It may therefore be worthwhile to focus attention on those gene regions that showed mid-range shifts in expression level in the treatment group when looking for evidence of adaptive evolution. In particular, the midrange transfrags related to metabolic and developmental processes as well as immune function are likely to be good candidates as genes of adaptive significance for increasing temperatures (Table 2). Rainbowfish represent ideal candidates for studies of local adaptation due to their reduced dispersal and distribution over multiple ecoregions. The suite of genes that we present here showing a response to increased temperature are a good starting point for further manipulative experiments or landscape wide surveys of genetic variation. Creating a catalogue of polymorphisms at these genes throughout the range of $M$. duboulayi will provide insights into the adaptive potential of this species in the face of a warming environment.

\section{RNA-seq recommendations for non-model taxa}

The results of this study highlight the appropriateness of an RNA-seq approach for studies of adaptation (including adaptive plasticity) in non-model organisms. With the paucity of genomic resources available for most wildlife species, NGS technologies offer the best hope for unravelling the processes of evolutionary adaptation in a natural setting. Rainbowfish are evolutionarily very different from their nearest genome-enabled species, Oryzias latipes, yet in this study we were able to generate a substantial list of candidate genes involved in a response to increasing temperatures. Over the past few years, the proliferation of software resources and validated pipelines for RNA-seq means that virtually any organism can now be the focus of ecological genomic research and this is reflected in the rapid increase in publications reporting RNA-seq analyses in non-human taxa. The limiting factors that remain now are bioinformatic expertise and incomplete reference data. Over half of the dysregulated transfrags identified in our study were unable to be identified or were of unknown function. This continues to be a major challenge for studies of ecological and evolutionary genomics [6]. Interpretation of genomic data lags well behind the current ability to generate that data. The limitation stems from the fact that annotation of genes of ecological interest still relies upon inferring homologies with genomic features established and developed in a few model species for non-ecological purposes. Better data integration is needed to facilitate the association of gene transcripts with specific natural conditions or phenotypic responses. Further work to characterise the function of these unknown genes via experimental studies of nonmodel organisms will enhance our understanding of the important biological pathways involved in responses to temperature stress and other environmental changes. We have shown that differing mapping and DE analysis approaches lead to very different outcomes in terms of the DE genes identified. While a combination of all available approaches is preferable to identify overlap in the candidate genes detected, we found that combining output from just Bowtie mapping and DESeq significance testing with BWA mapping and DESeq significance testing delivered just 21 more DE genes than combining all four approaches tested in our study (see Figure 1). This conservative approach is an efficient way to avoid large numbers of false positives being detected in RNA-seq studies.

\section{Conclusions}

Temperature increases predicted over the coming decades suggests species with limited dispersal abilities will need substantial adaptive potential to avoid extinction. That adaptive potential will likely come from a number of sources including adaptive phenotypic plasticity, standing genetic variation, and newly-derived mutations. Regardless of the source, adaptation will be most important in those processes related to heat tolerance. We 
have presented a first insight into which processes are likely to be important in the rainbowfish, $M$. duboulayi. This provides a foundation for future research into temperature-driven adaptive responses in freshwater species but also invites more detailed study of the phenome-genome interaction under conditions of temperature stress.

We identified a predictable suite of heat shock genes that responded sharply to increased temperatures in the treatment group. However, we also identified transfrags related to regulation of metabolic functions and developmental processes that showed mid-range levels of dysregulation and may be stronger candidates as genes for long-term adaptation to a warming environment. We present these candidate genes as targets for ongoing research into populations representing different thermal environments throughout the species range. We also expect that these candidates will be useful targets for studies of other freshwater species experiencing long-term thermal challenges.

The expression level changes we have presented may be an example of a plastic response. To check for an adaptive component it is necessary to repeat the temperature trial on other geographically distant populations and/or sister taxa. Parallel expression level changes in these populations would indicate plasticity whereas altered responses would be suggestive of adaptation at the genome level. Such "common garden" experiments allow the disentangling of pure plastic vs. genetic responses and are ideal approaches for future research. Other avenues to explore evolutionary adaptation to increased temperatures include investigating if DNA polymorphisms are present within and between populations at the gene regions we have identified in this study. Extensions of this research to include adaptive traits from other important environmental impacts will enable a much broader understanding of how freshwater species are likely to cope with human-induced habitat and climatic change.

\section{Availability of supporting data}

Raw sequencing data is available through the NCBI Sequence Read Archive under Project ID PRJNA205235 (http://trace.ncbi.nlm.nih.gov/Traces/sra/). All samples were sequenced as $101 \mathrm{bp}$ paired-end reads on an Illumina HiSeq2000 sequencer.

\section{Additional file}

Additional file 1: Table S1. Sequencing statistics for individual paired end reads from the pooled RNA-Seq library from $M$. duboulayi sequenced in a single lane of the Illumina HiSeq 2000. Table S2a. Annotated genes matching up-regulated transfrags in the high temperature group of M. duboulayi. Mean similarity is computed as the average similarity value for all the hits of a given sequence. Gene ontology abbreviations: $\mathrm{P}=$ biological process, $\mathrm{F}=$ molecular function, $\mathrm{C}=$ cellular component. Table S2b. Annotated genes matching down-regulated transfrags in the high temperature group of $M$. duboulayi. Mean similarity is computed as the average similarity value for all the hits of a given sequence.

\section{Competing interests}

The authors declare that they have no competing interests.

\section{Authors' contributions}

SS participated in the study planning and coordination, carried out the molecular genetic component, analysed the genomic data and drafted the manuscript. LBB conceived the study, participated in its design and coordination and helped to draft the manuscript. LB participated in the study design and planning and helped to draft the manuscript. All authors read and approved the final manuscript.

\section{Acknowledgments}

This study was funded by the Discovery Program of the Australian Research Council (ARC grant DP110101207 to L. Beheregaray and L. Bernatchez). We thank Leo O'Reilly and Simon Westergaard for assistance with sampling and fish husbandry, respectively. Animal ethical approval was received from Flinders University (AWC E342).

\section{Author details}

${ }^{1}$ Molecular Ecology Laboratory, School of Biological Sciences, Flinders University, Adelaide, SA 5001, Australia. ${ }^{2}$ Institut de Biologie Intégrative et des Systèmes, Université Laval, Québec, QC G1V 0A6, Canada. ${ }^{3}$ Current address: Department für Integrative Biologie und Evolution, Veterinärmedizinische Universität Wien, Vienna 1160, Austria.

Received: 29 January 2013 Accepted: 27 May 2013

Published: 5 June 2013

\section{References}

1. Shi Y, Li J, Jin Z: Advances in ecological genomics. Shengtai Xuebao/ Acta Ecologica Sinica 2012, 32(18):5846-5858.

2. Ungerer MC, Johnson LC, Herman MA: Ecological genomics: understanding gene and genome function in the natural environment. Heredity 2007, 100(2):178-183.

3. Ekblom R, Galindo J: Applications of next generation sequencing in molecular ecology of non-model organisms. Heredity 2011, 107(1):1-15.

4. Hornett EA, Wheat CW: Quantitative RNA-Seq analysis in non-model species: assessing transcriptome assemblies as a scaffold and the utility of evolutionary divergent genomic reference species. BMC Genomics 2012, 13(1):361.

5. Vijay N, Poelstra JW, Künstner A, Wolf JBW: Challenges and strategies in transcriptome assembly and differential gene expression quantification. Mol Ecol: A comprehensive in silico assessment of RNA-seq experiments; 2012.

6. Pavey SA, Bernatchez L, Aubin-Horth N, Landry CR: What is needed for next-generation ecological and evolutionary genomics? Ecology and Evolution 2012, 27(12):673-678.

7. de Ong W, Voo LYC, Kumar VS: De Novo Assembly, Characterization and Functional Annotation of Pineapple Fruit Transcriptome through Massively Parallel Sequencing. PLoS One 2012, 7(10):e46937.

8. Gagnaire PA, Normandeau E, Cote C, Hansen MM, Bernatchez L: The Genetic Consequences of Spatially Varying Selection in the Panmictic American Eel (Anguilla rostrata). Genetics 2012, 190(2):725-U703.

9. Gao X, Han J, Lu Z, Li Y, He C: Characterization of the spotted seal Phoca largha transcriptome using Illumina paired-end sequencing and development of SSR markers. Comp Biochem Physiol Part D Genomics Proteomics 2012, 7(3):277-284.

10. Garcia TI, Shen Y, Crawford D, Oleksiak MF, Whitehead A, Walter RB: RNASeq reveals complex genetic response to deepwater horizon oil release in Fundulus grandis. BMC Genomics 2012, 13(1):474.

11. Liu M, Qiao G, Jiang J, Yang H, Xie L, Xie J, Zhuo R: Transcriptome Sequencing and De Novo Analysis for Ma Bamboo (Dendrocalamus latiflorus Munro) Using the Illumina Platform. PLoS One 2012, 7(10):e46766. 
12. Miller HC, Biggs PJ, Voelckel C, Nelson NJ: De novo sequence assembly and characterisation of a partial transcriptome for an evolutionarily distinct reptile, the tuatara (Sphenodon punctatus). BMC Genomics 2012, 13(1):439.

13. St-Cyr J, Derome N, Bernatchez L: The transcriptomics of life-history trade-offs in whitefish species pairs (Coregonus sp.). Mol Ecol 2008, 17(7):1850-1870.

14. Cooke G, Chao N, Beheregaray L: Natural selection in the water: freshwater invasion and adaptation by water colour in the Amazonian pufferfish. J Evol Biol 2012, 25(7):1305-1320.

15. Lindquist S, Craig E: The heat-shock proteins. Annu Rev Genet 1988, 22(1):631-677.

16. Pipkin S, Rhodes C, Williams N: Influence of temperature on Drosophila alcohol dehydrogenase polymorphism. J Hered 1973, 64(4):181-185.

17. Crawford DL, Powers DA: Molecular basis of evolutionary adaptation at the lactate dehydrogenase-B locus in the fish Fundulus heteroclitus. Proc Natl Acad Sci 1989, 86(23):9365-9369.

18. Kassahn KS, Caley MJ, Ward AC, Connolly AR, Stone G, Crozier RH: Heterologous microarray experiments used to identify the early gene response to heat stress in a coral reef fish. Mol Ecol 2007, 16(8):1749-1763.

19. Lewis JM, Hori TS, Rise ML, Walsh PJ, Currie S: Transcriptome responses to heat stress in the nucleated red blood cells of the rainbow trout (Oncorhynchus mykiss). Physio/ Genomics 2010, 42(3):361-373.

20. Long Y, Li L, Li Q, He X, Cui Z: Transcriptomic Characterization of Temperature Stress Responses in Larval Zebrafish. PLoS One 2012, 7(5):e37209.

21. Buckley BA, Gracey AY, Somero GN: The cellular response to heat stress in the goby Gillichthys mirabilis: A cDNA microarray and protein-level analysis. J Exp Biol 2006, 209(14):2660-2677.

22. Logan CA, Somero GN: Transcriptional responses to thermal acclimation in the eurythermal fish Gillichthys mirabilis (Cooper 1864). Am J Physiol Regul Integr Comp Physiol 2010, 299(3):R843-R852.

23. Logan CA, Somero GN: Effects of thermal acclimation on transcriptional responses to acute heat stress in the eurythermal fish Gillichthys mirabilis (Cooper). Am J Physiol Regul Integr Comp Physiol 2011, 300(6):1373-1383.

24. Quinn NL, McGowan CR, Cooper GA, Koop BF, Davidson WS: Identification of genes associated with heat tolerance in arctic charr exposed to acute thermal stress. Physiol Genomics 2011, 43(11):685-696.

25. Buckley BA, Place SP, Hofmann GE: Regulation of heat shock genes in isolated hepatocytes from an Antarctic fish. Trematomus bernacchii. J Exp Biol 2004, 207(21):3649-3656.

26. Buckley BA, Hofmann GE: Thermal acclimation changes DNA-binding activity of heat shock factor 1 (HSF1) in the goby Gillichthys mirabilis: Implications for plasticity in the heat-shock response in natural populations. J Exp Biol 2002, 205(20):3231-3240.

27. West-Eberhard MJ: Phenotypic Plasticity and the Origins of Diversity Annu Rev Ecol Evol Syst 1989, 20:249-278. ArticleType: research-article / Ful publication date: 1989 / Copyright @ 1989 Annual Reviews.

28. Cheviron ZA, Whitehead A, Brumfield RT: Transcriptomic variation and plasticity in rufous-collared sparrows (Zonotrichia capensis) along an altitudinal gradient. Mol Ecol 2008, 17(20):4556-4569.

29. Allen GR, Midgley SH, Allen M: Field quide to the freshwater fishes of. Western Australian Museum: Australia; 2002.

30. Hattori A, Warburton K: Microhabitat use by the rainbowfish, Melanotaenia duboulayi, in a subtropical Australian stream. J Ethol 2003, 21(1):15-22.

31. McGuigan K, Zhu D, Allen GR, Moritz C: Phylogenetic relationships and historical biogeography of melanotaeniid fishes in Australia and New Guinea. Mar Freshwat Res 2000, 51(7):713-723.

32. Brown C: Do female rainbowfish (Melanotaenia spp.) prefer to shoal with familiar individuals under predation pressure? J Ethol 2002, 20(2):89-94.

33. Holdway DA, Hefferman J, Smith A: Multigeneration assessment of nonylphenol and endosulfan using a model Australian freshwater fish. Melanotaenia fluviatilis. Environ Toxicol 2008, 23(2):253-262.

34. Ferris R, Wilson RS: The physiological arms race: Exploring thermal acclimation among interacting species. J Therm Biol 2012, 37(3):236-242.

35. McCairns R, Bernatchez $L$ : Landscape genetic analyses reveal cryptic population structure and putative selection gradients in a large-scale estuarine environment. Mol Ecol 2008, 17(17):3901-3916

36. Young MJ: The efficacy of the aquatic anaesthetic AQUI-S ${ }^{\circledR}$ fo anaesthesia of a small freshwater fish. Melanotaenia australis. J Fish Biol 2009, 75(7):1888-1894

37. Flanagan SW, Ryan AJ, Gisolfi CV, Moseley PL: Tissue-specific HSP70 response in animals undergoing heat stress. Am J Physiol Regul Integr Comp Physiol 1995, 268(1):R28-R32.
38. Hall DM, Xu L, Drake VJ, Oberley LW, Oberley TD, Moseley PL, Kregel KC: Aging reduces adaptive capacity and stress protein expression in the liver after heat stress. J Appl Physio/ 2000, 89(2):749-759.

39. Kew M, Bersohn I, Seftel H, Kent G: Liver damage in heatstroke. Am J Med 1970, 49(2):192-202

40. Rabergh CM, Airaksinen S, Soitamo A, Bjorklund HV, Johansson T, Nikinmaa M, Sistonen L: Tissue-specific expression of zebrafish (Danio rerio) heat shock factor 1 mRNAs in response to heat stress. J Exp Biol 2000, 203(12):1817-1824

41. Giardine B, Riemer C, Hardison RC, Burhans R, Elnitski L, Shah P, Zhang Y, Blankenberg D, Albert I, Taylor J, et al: Galaxy: A platform for interactive large-scale genome analysis. Genome Res 2005, 15(10):1451-1455.

42. Schulz MH, Zerbino DR, Vingron M, Birney E: Oases: Robust de novo RNAseq assembly across the dynamic range of expression levels. Bioinformatics; 2012.

43. Surget-Groba Y, Montoya-Burgos Jl: Optimization of de novo transcriptome assembly from next-generation sequencing data. Genome Res 2010, 20(10):1432-1440

44. Langmead B, Trapnell C, Pop M, Salzberg S: Ultrafast and memory-efficient alignment of short DNA sequences to the human genome. Genome Biol 2009, 10(3):R25.

45. Li H, Durbin R: Fast and accurate short read alignment with BurrowsWheeler transform. Bioinformatics 2009, 25(14):1754-1760.

46. Li H, Handsaker B, Wysoker A, Fennell T, Ruan J, Homer N, Marth G, Abecasis G, Durbin R, Subgroup GPDP: The Sequence Alignment/Map format and SAMtools. Bioinformatics 2009, 25(16):2078-2079.

47. Anders S, Huber W: Differential expression analysis for sequence count data. Genome Biol 2010, 11(10):R106.

48. Conesa A, Götz S, Garcia-Gomez JM, Terol J, Talon M, Robles M: Blast2GO: a universal tool for annotation, visualization and analysis in functional genomics research. Bioinformatics 2005, 21:3674-3676.

49. Li H, Homer N: A survey of sequence alignment algorithms for nextgeneration sequencing. Brief Bioinform 2010, 11(5):473-483.

50. Robles JA, Qureshi SE, Stephen SJ, Wilson SR, Burden CJ, Taylor JM: Efficient experimental design and analysis strategies for the detection of differential expression using RNA-Sequencing. BMC Genomics 2012, 13(1):484.

51. Kvam VM, Liu P, Si Y: A comparison of statistical methods for detecting differentially expressed genes from RNA-seq data. Am J Bot 2012, 99(2):248-256

52. Airaksinen $\mathrm{S}$, Jokilehto $\mathrm{T}$, Råbergh $\mathrm{CM}$, Nikinmaa M: Heat- and coldinducible regulation of HSP70 expression in zebrafish ZF4 cells. Comp Biochem Physiol B Biochem Mol Biol 2003, 136(2):275-282.

53. Kassahn K, Crozier R, Ward A, Stone G, Caley MJ: From transcriptome to biological function: environmental stress in an ectothermic vertebrate, the coral reef fish Pomacentrus moluccensis. BMC Genomics 2007, 8(1):358.

54. Keller JM, Escara-Wilke JF, Keller ET: Heat stress-induced heat shock protein 70 expression is dependent on ERK activation in zebrafish (Danio rerio) cells. Comparative Biochemistry and Physiology Part A: Molecular \&amp; Integrative Physiology 2008, 150(3):307-314.

55. Krone PH, Lele Z, Sass JB: Heat shock genes and the heat shock response in zebrafish embryos. Biochem Cell Biol 1997, 75(5):487-497.

56. Murtha JM, Keller ET: Characterization of the heat shock response in mature zebrafish (Danio rerio). Exp Gerontol 2003, 38(6):683-691.

57. Quinn NL, McGowan CR, Cooper GA, Koop BF, Davidson WS: Ribosomal genes and heat shock proteins as putative markers for chronic, sublethal heat stress in arctic charr: Applications for aquaculture and wild fish. Physiol Genomics 2011, 43(18):1056-1064.

58. Fitzpatrick BM: Underappreciated consequences of phenotypic plasticity for ecological speciation. International Journal of Ecology 2012, 2012:Art. ID 25601.

59. Kültz D: Molecular and evolutionary basis of the cellular stress response. Annu Rev Physiol 2005, 67:225-257.

60. Thibert-Plante $X$, Hendry A: The consequences of phenotypic plasticity for ecological speciation. J Evol Biol 2011, 24(2):326-342.

doi:10.1186/1471-2164-14-375

Cite this article as: Smith et al:: RNA-seq analysis reveals extensive transcriptional plasticity to temperature stress in a freshwater fish species. BMC Genomics 2013 14:375. 